\title{
Multiscale damage simulation
}

\author{
Mario Timmel $^{1, *}$, Michael Kaliske ${ }^{2, * *}$, Stefan Kolling ${ }^{1, * * *}$, and Ralf Mueller $^{3, \dagger}$ \\ ${ }^{1}$ Daimler AG, Sindelfingen, Germany. \\ ${ }^{2}$ Institute for Structural Analysis, Dresden University of Technology, Germany. \\ ${ }^{3}$ Institute of Mechanics, Technical University of Darmstadt, Germany.
}

\begin{abstract}
Numerous materials show a softening behaviour at dynamic loading. The decrease of stress is caused by the evolution in the microscale in terms of areas where the local stiffness is reduced, e.g. due to micro-void growth. For a numerical treatment of this material behaviour, phenomenological damage approaches are used in daily engineering practice. For a better understanding of the micromechanical process of such phenomenological models, multiscale methods are becoming increasingly important. The physical quantities that are responsible for the microstructural evolution associated with the damage process are transferred into the numerical model. In this context, the method of configurational forces will be used to describe the geometrical changes of damaged areas. With the help of homogenization, macro- and microscale will be coupled. In consequence, each Gaussian point of the macroscale is modelled by an own microstructure (RVE), where the microscale evolves during the loading process according to observable damage phenomena. Hereby, we present the general case of hyperelastic materials at finite strains.
\end{abstract}

Copyright line will be provided by the publisher

\section{Configurational Forces in Two Phase Systems}

While the formulation of configurational forces for bulk materials can be obtained by the application of usual thermomechanical balance laws [5], equilibrium conditions have to be extended in the case of two phase systems because of the discontinuity of the field quantities [3]. At first, an arbitrary body $B$ is considered, where a soft inclusion $B^{-}$is embedded in a stiffer surrounding material $B^{+}$. Therefore, the strain energy $\Psi(\underline{\mathbf{F}}, \mathbf{X})$ depends in addition to the deformation gradient $\underline{\mathbf{F}}$ on the material position $\mathbf{X}$ with $\Psi^{-}(\mathbf{X}) \forall \mathbf{X} \in B^{-}$and $\Psi^{+}(\mathbf{X}) \forall \mathbf{X} \in B^{+}$and corresponds in general to the free energy $\Psi=\rho(\mathrm{u}-\eta \Theta)$, where $\mathrm{u}, \eta$ and $\Theta$ are the internal energy, the entropy density and the temperature. Based on the energy balance $\rho \llbracket \mathrm{u}+\frac{1}{2}|\dot{\mathbf{x}}|^{2} \rrbracket \mathrm{v}_{\mathrm{n}}+\llbracket(\underline{\mathbf{P n}}) \cdot \dot{\mathbf{x}} \rrbracket=0$ and the entropy balance $\rho \Theta \llbracket \eta \rrbracket \mathrm{v}_{\mathrm{n}}-\omega_{\mathrm{S}} \Theta=0$, the dissipation inequality for a two phase system is given by $\llbracket \Psi+0.5 \rho|\dot{\mathbf{x}}|^{2} \rrbracket \mathrm{v}_{\mathrm{n}}+\llbracket(\underline{\mathbf{P n}}) \cdot \dot{\mathbf{x}} \rrbracket=\omega_{\mathrm{S}} \Theta \geq 0$, where $\llbracket \cdot \rrbracket=(\cdot)^{+}-(\cdot)^{-}$denotes the jump of a quantity across the phase boundary $\mathrm{S}$. In the equations above, the quantities $\underline{\mathbf{P}}$ and $\dot{\mathrm{x}}$ represent the first Piola Kirchhoff stress and the velocity of material points. The material density $\rho=\rho^{+}=\rho^{-}$is assumed to be identical for $B^{+}$and $B^{-}$. According to the dissipation inequality, entropy $\omega_{\mathrm{S}}$ is produced if the phase boundary is moved in normal direction $\mathbf{n}$ with the velocity $\mathrm{v}_{\mathrm{n}}$. Using the momentum balance $\llbracket \underline{\mathbf{P}} \mathbf{n} \rrbracket+\rho \llbracket \dot{\mathbf{x}} \rrbracket \mathrm{v}_{\mathrm{n}}=\mathbf{0}$, the middle term of dissipation inequality can be reformulated as $\llbracket(\underline{\mathbf{P n}}) \cdot \dot{\mathbf{x}} \rrbracket=-\rho \mathrm{v}_{\mathrm{n}} \llbracket|\dot{\mathbf{x}}|^{2} \rrbracket-\mathrm{v}_{\mathrm{n}} \llbracket \underline{\mathbf{F}}^{\mathrm{T}} \underline{\mathbf{P}} \rrbracket \mathbf{n}-\rho \mathrm{v}_{\mathrm{n}}^{2} \llbracket \underline{\mathbf{F}}^{\mathrm{T}} \dot{\mathbf{x}} \rrbracket \cdot \mathbf{n}$ which leads to the modified dissipation inequality

$$
(\underbrace{\mathbf{n} \cdot \llbracket \underline{\mathbf{M}} \rrbracket \mathbf{n}-\mathrm{v}_{\mathrm{n}} \rho \mathbf{n} \cdot \llbracket \underline{\mathbf{F}}^{\mathrm{T}} \dot{\mathbf{x}} \rrbracket}_{\tau_{\mathrm{n}}}) \mathrm{v}_{\mathrm{n}}=\underbrace{\omega^{\mathrm{N}}}_{\text {d loc }_{\mathrm{S}}^{\omega_{\mathrm{S}} \Theta}} \geq 0 \quad \text { with } \quad \underline{\mathbf{M}}=\left(\Psi-0.5 \rho|\dot{\mathbf{x}}|^{2}\right) \underline{\mathbf{1}}-\underline{\mathbf{F}}^{\mathrm{T}} \underline{\mathbf{P}} .
$$

The quantity $\underline{\mathbf{M}}$ denotes the dynamic energy momentum tensor [2]. According to Equation (1), the local dissipation $\mathrm{d}_{\mathrm{S}}^{\text {loc }}$ is driven by the configurational force $\tau_{\mathrm{n}}$. The global dissipation has to be computed by integration over the phase boundary S. Here, a useful simplification of the procedure is to reach in the case of parametrization of S. In this context, the geometrical form of the inclusion is restricted to 3D-ellipsoids. These restrictions allow to model a macroscopical anisotropic damage characteristic and an analytical computation of $\tau_{\mathrm{n}}$ in the small strain case [1], [4]. An arbitrary ellipsoidal inclusion can be defined by a vector $\mathcal{X}=\mathcal{X}(\mathrm{m}, \mathrm{V}, \varphi)$. The quantities $\mathrm{V}, \mathrm{m}$ and $\varphi$ denote volume, aspect ratio $\mathrm{m}=(\mathrm{a}-\mathrm{b}) /(\mathrm{a}+\mathrm{b})$, where a and $\mathrm{b}$ are the semi-axes of the ellipsoid, and the rotation angle of the inclusion respectively. Consequently, the global dissipation inequality yields

$$
\mathcal{D}=\int_{\mathrm{S}} \tau_{\mathrm{n}}\left[\left(\partial_{\mathrm{m}} \mathcal{X} \cdot \mathbf{n}\right) \dot{\mathrm{m}}+\left(\partial_{\mathrm{V}} \mathcal{X} \cdot \mathbf{n}\right) \dot{\mathrm{V}}+\left(\partial_{\varphi} \mathcal{X} \cdot \mathbf{n}\right) \dot{\varphi}\right] \mathrm{dA}=\tau_{\mathrm{m}} \dot{\mathrm{m}}+\tau_{\mathrm{V}} \dot{\mathrm{V}}+\tau_{\varphi} \dot{\varphi} \geq 0
$$

where the quantities $\tau_{\mathrm{V}}, \tau_{\mathrm{m}}$ and $\tau_{\varphi}$ denote the consistent driving force with respect to the modification of volume, shape and rotation. To change the inclusion's configuration, relations $\dot{\mathrm{m}}=f\left(\tau_{\mathrm{m}}\right), \dot{\mathrm{V}}=f\left(\tau_{\mathrm{V}}\right)$ and $\dot{\varphi}=f\left(\tau_{\varphi}\right)$ have to be chosen that

\footnotetext{
* E-mail: mario.timmel@daimler.com, Phone: +4970319074573, Fax: +497113052 192203

** Corresponding author E-mail: michael.kaliske@tu-dresden.de, Phone: +4935146334386.

*** E-mail: stefan.kolling@daimler.com, Phone: +49703190 82829, Fax: +49711 3052136915.

† E-mail: r.mueller@mechanik.tu-darmstadt.de, Phone: +496151 164648, Fax: +496151 163018.
} 
satisfy the dissipation inequality (2). To fulfil the constraint $\tau_{\mathrm{V}} \dot{\mathrm{V}} \geq 0$, the inclusion's volume will be modified only in case of a new of $\tau_{\mathrm{V}}$ with $\mathrm{V}=\mathrm{V}_{0}+\left(\mathrm{V}_{\max }-\mathrm{V}_{0}\right)(1-\exp (\beta(t) / \xi))$ where $\beta(t)=\max _{s \in[0, t]}\left[\tau_{\mathrm{V}}(s) A(0)(A(s))^{-1}\langle\operatorname{tr} \underline{\mathbf{E}}\rangle(\operatorname{tr} \underline{\mathbf{E}})^{-1}\right]$ and $\xi, \mathrm{V}_{\max }$ and $\mathrm{V}_{0}$ are saturation parameters to control the rate of void growth, the maximum volume and the initial inclusion's volume, respectively. With $A(s)=\int_{\mathrm{S}(\mathrm{s})} \mathrm{dA}$, the driving force on the volume is regularized to avoid self growth of the voids at constant external loads due to a changing integration range. By using the convention of McCauley brackets for the trace of Green-Lagrange strain $\langle\operatorname{tr} \underline{\mathbf{E}}\rangle=0.5(\operatorname{tr} \underline{\mathbf{E}})+0.5|(\operatorname{tr} \underline{\mathbf{E}})|$, void growth at hydrostatic compression can be suppressed. The remaining dissipation inequality $\tau_{\mathrm{m}} \dot{\mathrm{m}}+\tau_{\varphi} \dot{\varphi} \geq 0$ can be fulfilled with $\dot{\mathrm{m}}=\alpha_{\mathrm{m}} \tau_{\mathrm{m}} \frac{V_{0}}{V}$ and $\dot{\varphi}=\alpha_{\varphi} \tau_{\varphi} \frac{V_{0}}{V}$, with the material parameters $\alpha_{\mathrm{m}} \geq 0$ and $\alpha_{\varphi} \geq 0$. The relation of $\tau_{\mathrm{m}}$ and $\tau_{\varphi}$ to the current volume $V$ is caused by a necessary regularization to uncouple the evolution in shape and rotation from the evolution in volume.

\section{Applications}

On the left hand side of Figure 1, the microstructures (edge-length $0.2 \mathrm{~mm}$, initial semiaxes of inclusion: $\mathrm{a}=\mathrm{b}=8.66 / 500 \mathrm{~mm}$ ) as well as the macrostructure $(\mathrm{b}=100 \mathrm{~mm}, \mathrm{~h}=20 \mathrm{~mm}$ ) after unloading are shown. The microstructure is constituted by Yeoh's model [6], where the material parameters for the inclusion (discretized with 216 solid-elements) as well as for the surrounding material (discretized with 1296 solid-elements) are $C_{1}^{-}=(1 / 10) C_{1}^{+}=1.0 \mathrm{~N} / \mathrm{mm}^{2}, C_{2}^{-}=(1 / 100) C_{2}^{+}=0.1 \mathrm{~N} / \mathrm{mm}^{2}$ and $C_{3}^{-/+}=0$. Based on homogenization procedure [4], the deformation state of the macroscale can be transferred to the microstructure, $\langle\underline{\mathbf{F}}\rangle=\frac{1}{V} \int_{B} \underline{\mathbf{F}}(\mathbf{X}) d V$. The loading is given by a deformation $u$ at the cantilever with a triangular $u$ - $t$-path. The evolution parameters are given by $\eta=0.5 \mathrm{~N} / \mathrm{mm}^{2}, \alpha_{m}=5.0$ and $\alpha_{\varphi}=5.0$. The microstructure belonging to the upper left macro-element evolves in shape and volume. The bottom microstructure evolves in shape only due to negative trace of stress. The intensity of evolution decreases to the right side of cantilever, where the initial and final configuration of the microscale coincide.

The quantitative effect of this evolution process is pointed out at the right hand side of Figure 1, where the macroscopic Cauchy stress is given by $\langle\underline{\boldsymbol{\sigma}}\rangle=(\operatorname{det}\langle\underline{\mathbf{F}}\rangle)^{-1}\langle\underline{\mathbf{F}}\rangle\left\langle\underline{\mathbf{P}}^{\mathrm{T}}\right\rangle$ with $\left\langle\underline{\mathbf{P}}^{\mathrm{T}}\right\rangle=\frac{1}{V} \int_{B} \underline{\mathbf{P}}^{\mathrm{T}}(\mathbf{X}) d V$. Due to the additional evolution in volume, the stress of the upper left RVE reduces more intensiv than the stress of the lower part. With the help of twoscale approaches, a better understanding and modeling of damage phenomena is possible. The presented procedure is not restricted to hyperelastic materials but can also be used for arbitrary materials.
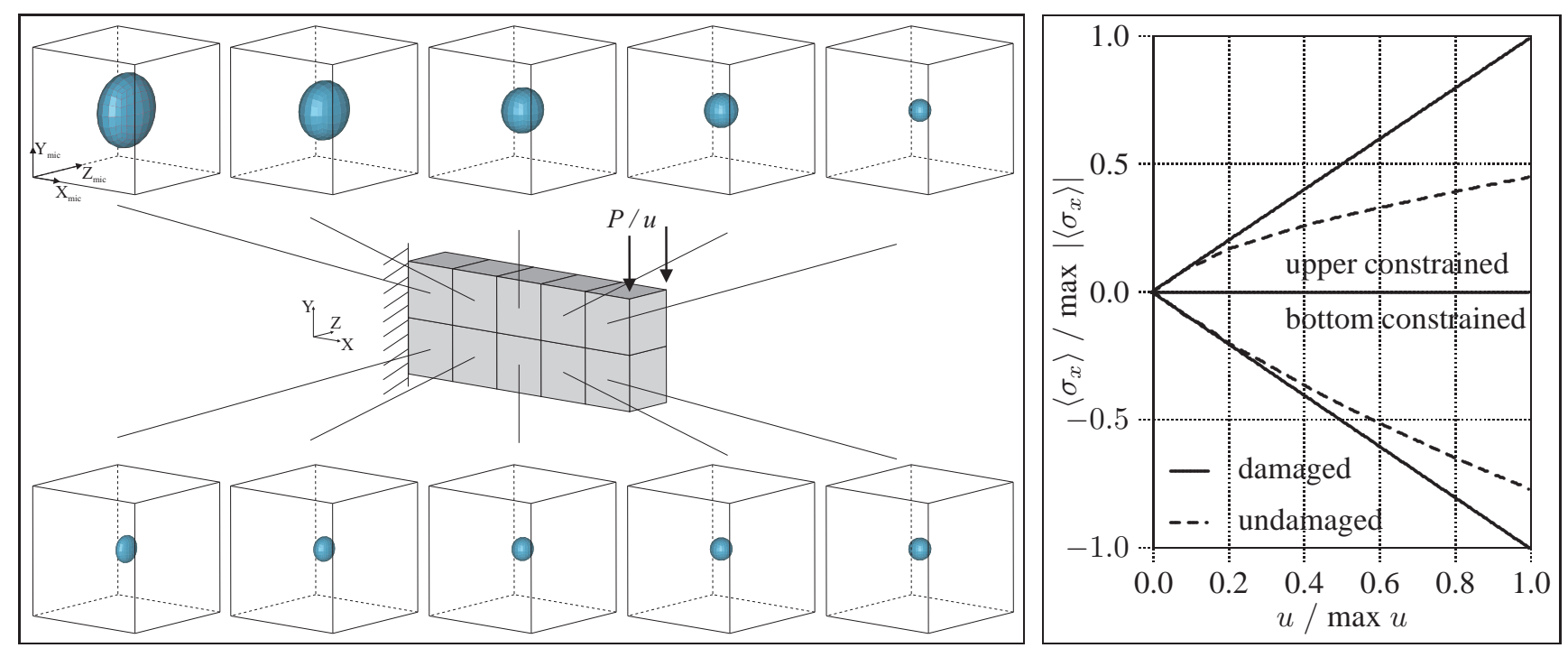

Fig. 1 Quantitative (left) and qualitative (right) results of twoscale-FEM-simultion

\section{References}

[1] J. D. Eshelby, Proc. Royal Society of London 241, 376-396 (1957).

[2] J. D. Eshelby, In Kanninen: Inelastic behaviour of Solids pp. 87-112 (Mc Graw Hill, New York, 1970).

[3] M. E. Gurtin, Configurational forces as basic concept of continuum physics (Springer, Berlin, 2000).

[4] R. Mueller and D. Gross, Mechanics of Materials 22, 63-76 (2001).

[5] M. Timmel, M. Kaliske, S. Kolling and R. Mueller, Computers, Materials and Continua 5, 161-172 (2007).

[6] O. H. Yeoh, Rub. Chem. Techn., 63, 792-805 (1990). 